\title{
CFOA Based Filter Design Circuit a New Configuration
}

\author{
Praween Kumar Sinha ${ }^{1}$, Abhishek Vikram ${ }^{2}$, Kalyan Singh Yadav ${ }^{3}$ \\ ${ }^{1}$ Assistant Professor, Dept. of ECE, MAIT, New Delhi, India. \\ ${ }^{2}$ Digital Design Teacher, BodhiSattva Educational Trust, New Delhi, India. \\ ${ }^{3} H O D$, Dept. of ECE, NIEC, New Delhi, India.
}

\begin{abstract}
In this paper, CFOA based filters are designed and simulated. Filters are electronic circuits typically used for conditioning. As traditional filters consist of passive elements which require larger chip area and consumes more power. Those passive elements are replaced by active component CFOA which fulfills several purposes with improved slew rate, gain bandwidth and many. The values for the passive components are calculated, and the circuits are then simulated with ICAP to reach the final conclusion which will describe the results of the simulations compared to theoretical results. ${ }^{[3]}$
\end{abstract}

Keywords : CFOA (Current Feedback Operational Amplifier)

\section{Introduction}

The current feedback operational amplifier otherwise known as CFOA is a type of electronic amplifier whose inverting input is sensitive to current, rather than to voltage as in a conventional VFA. CFA do not have the traditional differential amplifier input structure, thus they sacrifice the parameter matching inherent to that structure.

The current feedback operational amplifier (CFOA) [1] is a suitable active block for synthesis of filters for higher frequency domain. When the part with available compensation pin is mentioned, there are too many options to create some new circuits. The design based on synthesis of general filter circuits [2] is a good way to obtain acceptable results. The use of two amplifiers has an advantage, because parameters of a final circuit can be usually set independent.

The transfer function was calculate and expressed for every input-output combination. The terms which exactly match the standards of typical filters were considered as correct. Standards, namely low-pass, high-pass, and band pass are defined as follows:

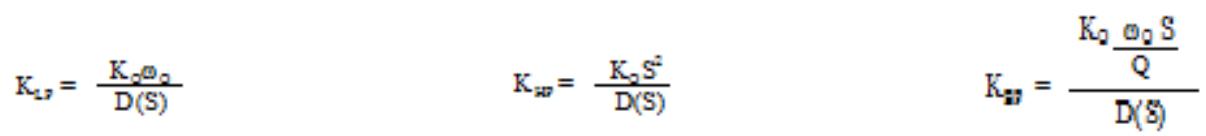

\section{CFA Symbol}

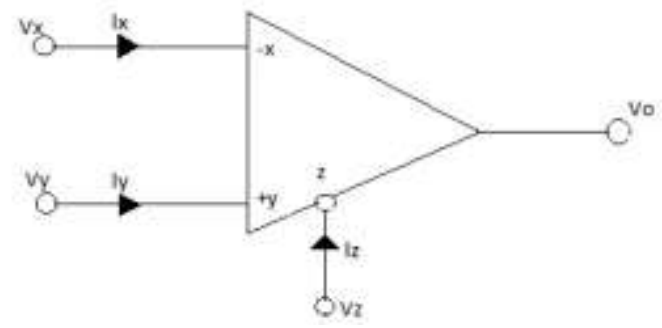

\section{Terminal Equations of CFA}
1. $\mathrm{Iy}=0$
2.
$\mathrm{Ix}=\mathrm{Iz}$
3. $\quad V x=V y$
4. $\quad \mathrm{Vz}=\mathrm{Vo}$

It is necessary to take into account a restriction in this field of circuit design. There are few types of mentioned CFOA with compensational pin, but just one of them is currently available for practical design. It is the amplifier AD844 [7] produced by Analog Devices with trans-impedance $3 \mathrm{M} \Omega$ and with relatively narrow declared gain bandwidth 60MHz. other devices, e.g. AD846 and HFA1102, are marked as obsolete.

\section{General Circuit for Low Pass Filter}

The new circuit with two CFOAs and four admittances was intuitively compiled. The new configured circuit diagram is given next 


\subsection{Circuit Diagram}

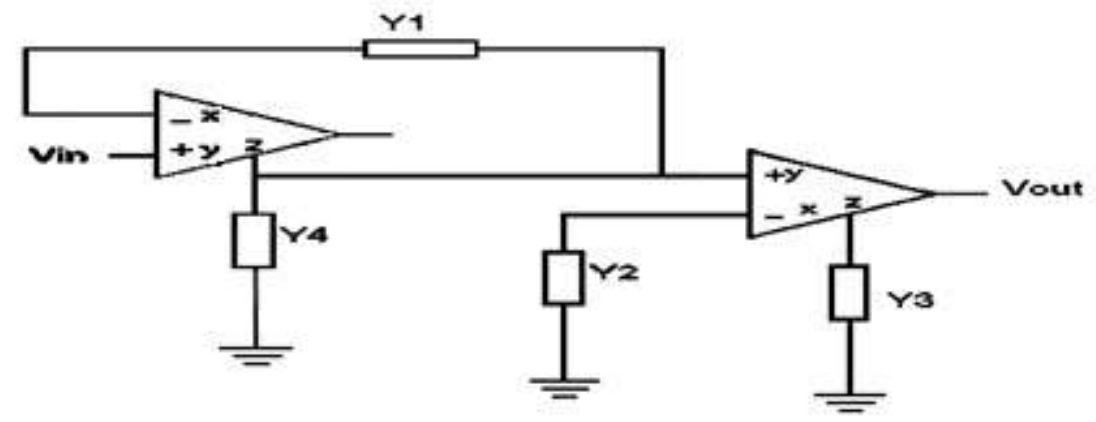

Fig. Low Pass falter using CFOA

The calculation of characteristic equation (transfer function) is given below:

$\mathrm{V}_{\text {in }}=$ Input Signal

$\mathrm{V}_{\mathrm{o} 1}=$ Output signal Of first CFOA

$\mathrm{V}_{\mathrm{o} 2}=$ Output Signal of second CFOA

$\left(0-\mathrm{V}_{\mathrm{o} 1}\right) \mathrm{Y}_{2}=\left(0-\mathrm{V}_{\mathrm{o} 2}\right) \mathrm{Y}_{3}$

$\left(0-\mathrm{V}_{\mathrm{o} 1}\right) \mathrm{Y}_{4}+\left(\mathrm{V}_{\text {in }}-\mathrm{V}_{\mathrm{o} 1}\right) \mathrm{Y}_{1}=\left(\mathrm{V}_{\mathrm{o} 1}-\mathrm{V}_{\text {in }}\right) \mathrm{Y}_{1}$

$\mathrm{V}_{\mathrm{o} 1}=2 \mathrm{Y} 1 . \mathrm{Vin} / \mathrm{Y}_{4}+2 \mathrm{Y}_{1}$

From (i) and (ii) we get

$-\left(2 \mathrm{Y}_{1} \cdot \mathrm{Vin} / \mathrm{Y}_{4}+2 \mathrm{Y}_{1}\right) \mathrm{Y}_{2}=\mathrm{V}_{\mathrm{o} 2} . \mathrm{Y}_{3}$

$\mathrm{V}_{\mathrm{o} 2}=2 \mathrm{Y}_{1} \cdot \mathrm{Y}_{2} \cdot \mathrm{V}_{\text {in }} / \mathrm{Y}_{3}\left(\mathrm{Y}_{4}+2 \mathrm{Y}_{1}\right)$

The individual admittances are defined as follows

$\mathrm{Y}_{1}=\mathrm{G}_{1}$ and $\mathrm{Y}_{2}=\mathrm{G}_{2}, \quad \mathrm{Y}_{3}=\mathrm{SC}_{3}+\mathrm{G}_{3}$ and $\mathrm{Y}_{4}=\mathrm{SC}_{4}$

Applying this in above equation we get,

$\mathrm{V}_{\mathrm{o} 1} / \mathrm{V}_{\text {in }}=2 \mathrm{G} 1 . \mathrm{G}_{2} /\left(\left[\mathrm{Sc}_{3}+\mathrm{G}_{3}\right]\left[\mathrm{SC}_{4}+2 \mathrm{G}_{1}\right]\right)$

The characteristic equation is

$T(s)=V_{\text {out }} / V_{\text {in }}=V_{02} / V_{\text {in }}=2 G_{1} \cdot G_{2} /\left(S^{2} \cdot C_{3} \cdot C_{4}+S\left(2 G_{1} \cdot C_{3}+C 4 \cdot G_{3}\right)+2 G_{1} \cdot G_{3}\right.$

$\omega_{0}=\sqrt{(}\left(2 \mathrm{G}_{1} \cdot \mathrm{G}_{3} / \mathrm{C}_{3} \cdot \mathrm{C}_{4}\right)$

$\mathrm{G}_{1}=1 / \mathrm{R}_{1}=1 / 470 \Omega=2.127 * 10^{-3}$

$\mathrm{G}_{3}=1 / \mathrm{R}_{3}=1 / 10^{3} \quad \mathrm{C}_{3}=0.01 \mu \mathrm{f}$

$\mathrm{C}_{4}=0.01 \mu \mathrm{f}$

$\omega_{0}=2.062 * 10^{5}$

Cut-off frequency for the filter is calculated as

$\mathbf{f}_{\mathbf{o}}=\omega_{\mathrm{o}} / \mathbf{2} \boldsymbol{\tau}=\mathbf{3 2 . 8} \mathrm{KHz}$

The result of simulation i.e. the schematic diagram and resulted waveform is given below

\subsection{Schematic Diagram}

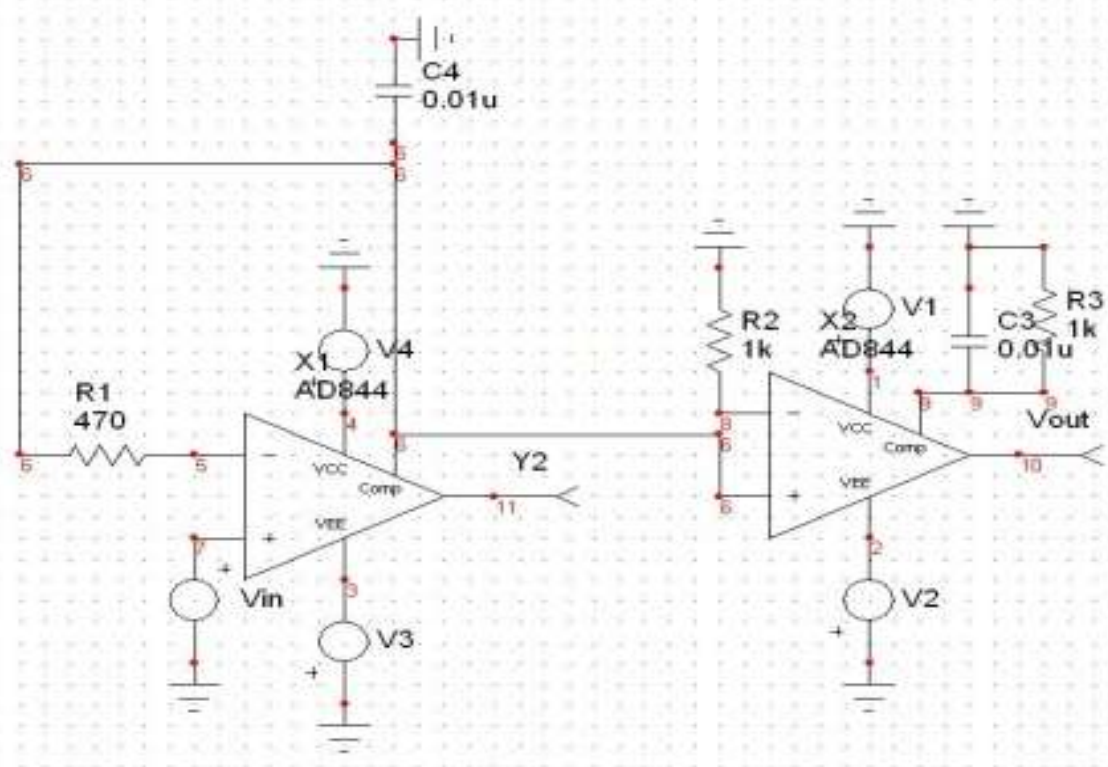




\section{General Circuit for High Pass Filter}

The new circuit with two CFOAs and four admittances was intuitively compiled. The new configured circuit diagram is given next

\subsection{Circuit Diagram}

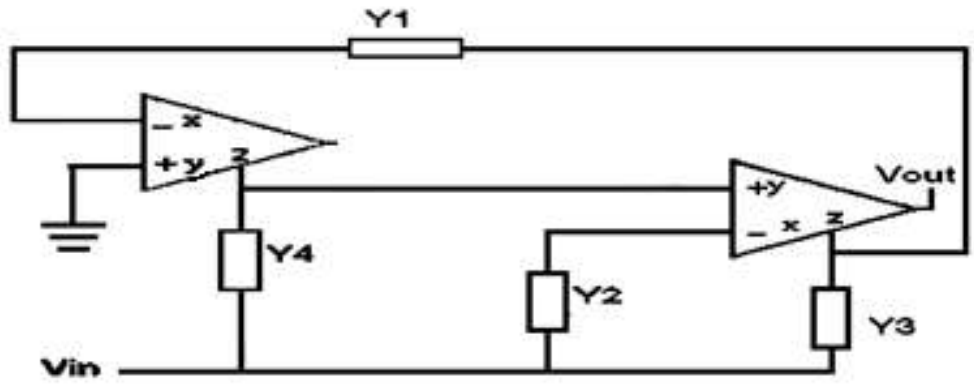

Fig. High Pass filter using CFOA

The calculation of characteristic equation (transfer function) is given below:

$\mathrm{V}_{\text {in }}=$ Input Signal

$\mathrm{V}_{\mathrm{o} 1}=$ Output signal Of first CFOA

$\mathrm{V}_{\mathrm{o} 2}=$ Output Signal of second CFOA

$\left(\mathrm{V}_{\mathrm{in}}-\mathrm{V}_{\mathrm{o} 1}\right) \mathrm{Y}_{4}=\left(\mathrm{V}_{\mathrm{o} 2}-0\right) \mathrm{Y}_{1}$

$\left(\mathrm{V}_{\mathrm{in}}-\mathrm{V}_{\mathrm{o} 1}\right) \mathrm{Y}_{4}=\mathrm{V}_{\mathrm{o} 2} \mathrm{Y}_{1}$

$\left(\mathrm{V}_{\text {in }}-\mathrm{V}_{\mathrm{o} 2}\right) \mathrm{Y}_{3}=\left(\mathrm{V}_{\mathrm{o} 2}-0\right) \mathrm{Y}_{1}+\left(\mathrm{V}_{\mathrm{in}}-\mathrm{V}_{\mathrm{o} 1}\right) \mathrm{Y}_{2}$

$\mathrm{V}_{\text {o1 }} / \mathrm{V}_{\text {in }}=\left[\mathrm{Y}_{1} \mathrm{Y}_{2}+\mathrm{Y}_{4}\left(\mathrm{Y}_{1}+\mathrm{Y}_{3}\right)-\mathrm{Y}_{1}\right] /\left[\mathrm{Y}_{1} \mathrm{Y}_{2}+\mathrm{Y}_{4}\left(\mathrm{Y}_{1}+\mathrm{Y}_{3}\right)\right]$

Now using equation (i) in eq (ii)

$\left(\mathrm{V}_{\text {in }}-\mathrm{V}_{\mathrm{o} 2}\right) \mathrm{Y}_{3}=\mathrm{V}_{\mathrm{o} 2} \mathrm{Y}_{1}+\mathrm{V}_{\text {in }} \mathrm{Y}_{2}-\left[\mathrm{Y} 4 . \mathrm{V}_{\text {in }}-\mathrm{V}_{\mathrm{o} 2} \mathrm{Y}_{1}\right] \mathrm{Y}_{2} / \mathrm{Y}_{4}$

The characteristic equation obtained is

$\mathbf{V}_{\text {o2 }} / \mathbf{V}_{\text {in }}=S^{2} /\left(S^{2}+S G / C_{3}+G_{1} G_{2} / C_{3} C_{4}\right)$

$\omega_{0}=\sqrt{ }\left(\mathrm{G}_{1} \cdot \mathrm{G}_{2} / \mathrm{C}_{3} \cdot \mathrm{C}_{4}\right)$

$\mathrm{G}_{1}=1 / \mathrm{R}_{1}=1 / 470 \Omega=2.127 * 10^{-3}$

$\mathrm{G}_{3}=1 / \mathrm{R}_{3}=1 / 10^{3}$

$\mathrm{C}_{3}=0.01 \mu \mathrm{f}$

$\mathrm{C}_{4}=0.01 \mu \mathrm{f}$

$\omega_{0}=\sqrt{ }\left(2127 * 10^{6}\right)$

Cut-off frequency for the filter is calculated as

$f_{o}=\omega o / 2 \pi=23.23 \mathrm{KHz}$

The result of simulation i.e. the schematic diagram and resulted waveform is given below

\subsection{Schematic Diagram}

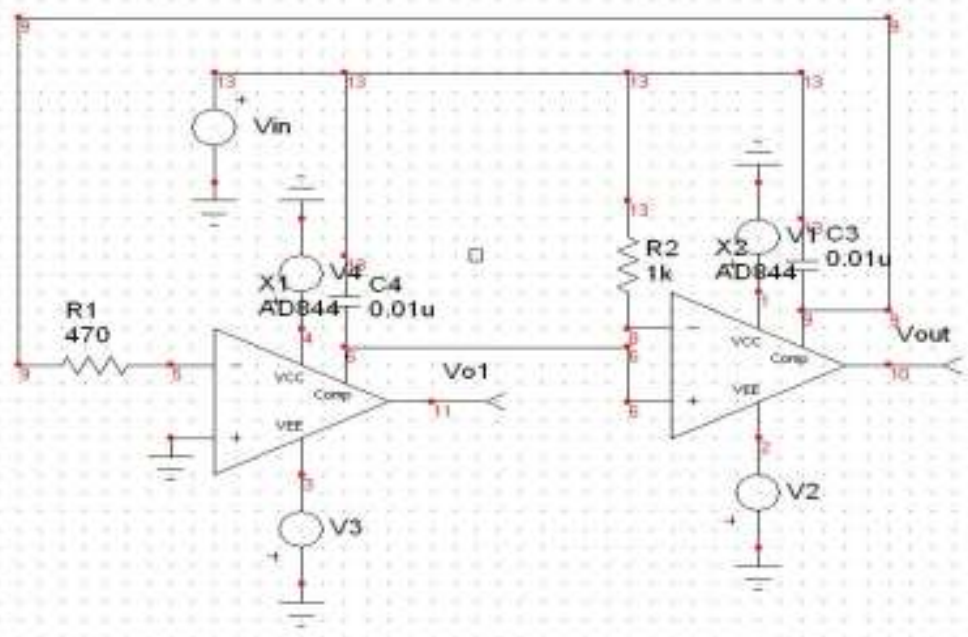




\section{General Circuit For Band Pass Filter}

The new circuit with two CFOAs and four admittances was intuitively compiled. The new configured circuit diagram is given next

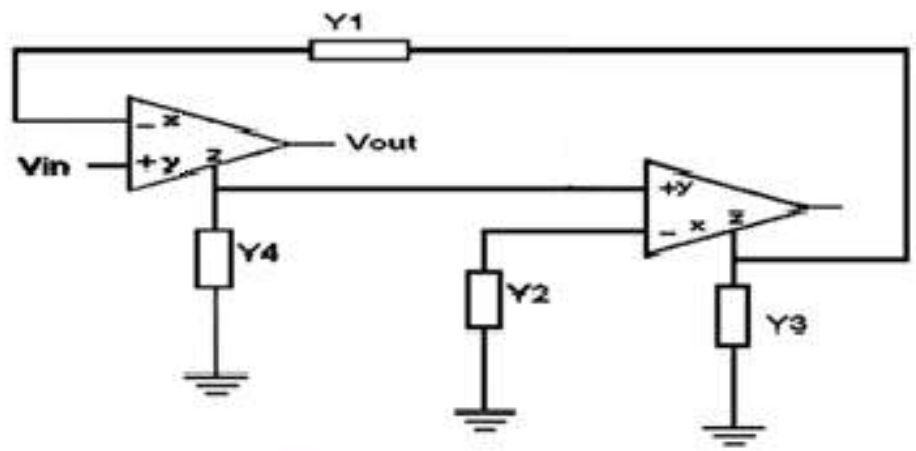

Fig. Band Pass fiter using CFOA

The calculation of characteristic equation (transfer function) is given below:

$\mathrm{V}_{\text {in }}=$ Input Signal

$\mathrm{V}_{\mathrm{o} 1}=$ Output signal of first CFOA

$\mathrm{V}_{\mathrm{o} 2}=$ Output Signal of second CFOA

$\left(0-\mathrm{V}_{\mathrm{o} 1}\right) \mathrm{Y}_{4}=\left(\mathrm{V}_{\mathrm{o} 2}-\mathrm{V}_{\mathrm{in}}\right) \mathrm{Y}_{1}$

$\mathrm{V}_{\mathrm{o} 1}=-\left(\mathrm{V}_{\mathrm{o} 2}-\mathrm{V}_{\mathrm{in}}\right) \mathrm{Y}_{1} / \mathrm{Y}_{4}$

$\left(0-\mathrm{V}_{\mathrm{o} 1}\right) \mathrm{Y}_{2}+\left(\mathrm{V}_{\mathrm{o} 2}-\mathrm{V}_{\mathrm{in}}\right) \mathrm{Y}_{1}=\left(0-\mathrm{V}_{\mathrm{o} 2}\right) \mathrm{Y}_{3}$

Putting $\mathrm{V}_{\mathrm{o} 1}$ in eq (iii)

$\mathrm{V}_{\mathrm{o} 2}=\left(\mathrm{V}_{\text {in }} \mathrm{Y}_{1}-\mathrm{V}_{\mathrm{o} 1} \mathrm{Y}_{4}\right) / \mathrm{Y}_{1}$

Putting eq (iv) in eq (iii)

$\mathrm{V}_{\mathrm{o} 1} \mathrm{Y}_{2}+\mathrm{V}_{\text {in }} \mathrm{Y}_{1}=\left[\mathrm{V}_{\text {in }} \mathrm{Y}_{1}-\mathrm{V}_{\mathrm{o} 1} \mathrm{Y}_{4}\right]\left[\mathrm{Y}_{1}+\mathrm{Y}_{3}\right]$

The characteristic equation calculated is

$\mathbf{V}_{\text {o1 }} / \mathbf{V}_{\text {in }}=\mathbf{Y}_{1} \mathbf{Y}_{3} /\left(\mathbf{Y}_{1} \mathbf{Y}_{2}+\mathbf{Y}_{4}\left(\mathbf{Y}_{1}+\mathbf{Y}_{3}\right)\right)$

The individual admittances are as follows

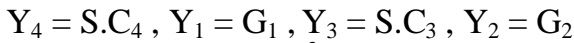

$\mathrm{V}_{\mathrm{o} 1} / \mathrm{V}_{\mathrm{in}}=\left(\mathrm{G}_{1} / \mathrm{C}_{4} \mathrm{~S}\right) /\left(\mathrm{S}^{2}+\mathrm{S}_{\mathrm{G}} \mathrm{G}_{1} / \mathrm{C}_{3}+\mathrm{G}_{1} \mathrm{G}_{2} / \mathrm{C}_{3} \mathrm{C}_{4}\right)$

$\omega_{0}=\sqrt{ }\left(\mathrm{G}_{1} \cdot \mathrm{G}_{2} / \mathrm{C}_{3} \cdot \mathrm{C}_{4}\right)$

$\mathrm{G}_{1}=1 / \mathrm{R}_{1}=1 / 470 \Omega=2.127 * 10^{-3}$

$\mathrm{G}_{3}=1 / \mathrm{R}_{3}=1 / 10^{3} \quad \mathrm{C}_{3}=0.01 \mu \mathrm{f}$

$\mathrm{C}_{4}=0.01 \mu \mathrm{f}$

$\omega_{0}=\sqrt{ }\left(2127 * 10^{6}\right)$

Cut-off frequency and bandwidth calculated is

$f_{o}=\omega o / 2 \pi=23.23 \mathrm{KHz}$

Bandwidth $=\omega_{\mathrm{o}} / \mathbf{Q}=\mathbf{G}_{1} / \mathbf{C}_{3}=\mathbf{C}_{3} \cdot \mathbf{R}_{1}$

$$
=33.8 \mathrm{KHz}
$$

\subsection{Schematic Diagram}

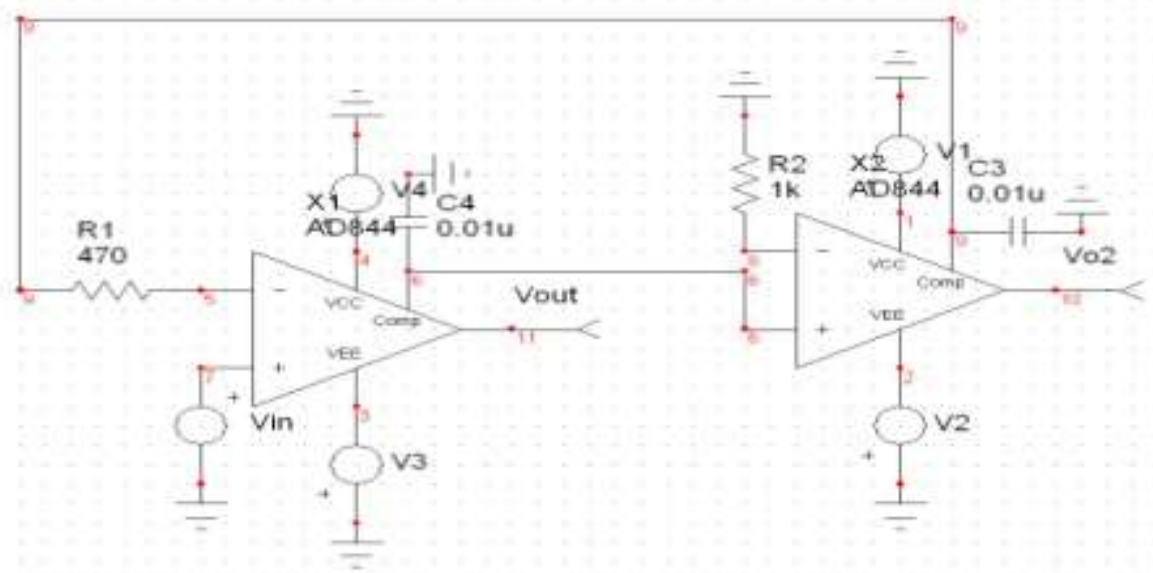


Figures And Tables
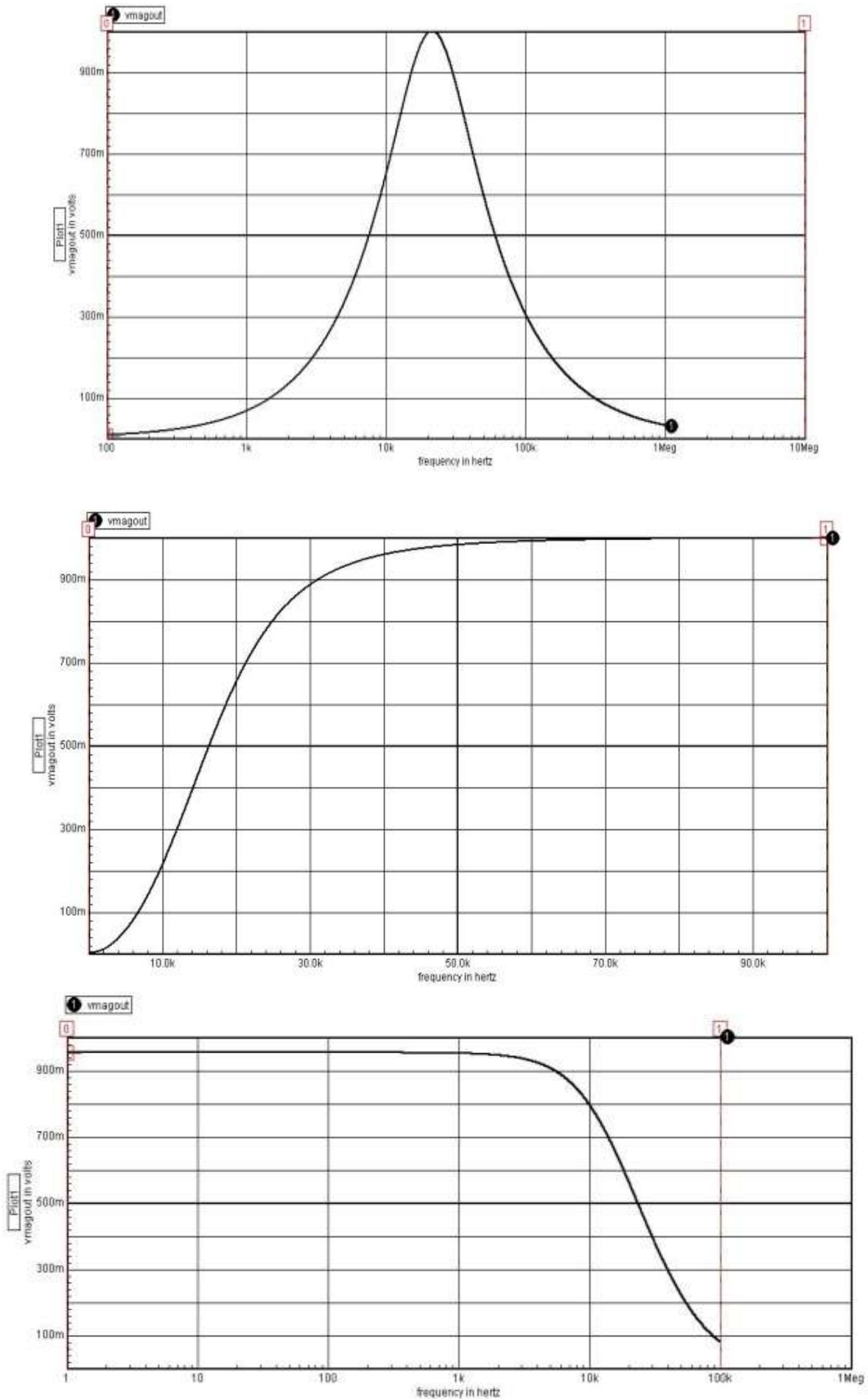

\begin{tabular}{|c|c|}
\hline Type of filter & \multicolumn{1}{|c|}{$\begin{array}{c}\text { Transfer Function } \\
\mathbf{T}(\mathbf{s})\end{array}$} \\
\hline Low Pass filter & $\mathbf{G}_{2} /\left(\mathbf{S}^{2} \cdot \mathbf{C}_{3} \cdot \mathbf{C}_{4}+\mathbf{S}\left(\mathbf{2} \mathbf{G}_{1} \cdot \mathbf{C}_{3}+\mathbf{C}_{4} \cdot \mathbf{G}_{3}\right)+2 \mathbf{G}_{1} \cdot \mathbf{G}_{3}\right.$ \\
\hline High Pass Filter & $\mathbf{S}^{2} /\left(\mathbf{S}^{2}+\mathbf{S G} / \mathbf{C}_{3}+\mathbf{G}_{1} \mathbf{G}_{2} \mathbf{C}_{3} \mathbf{C}_{4}\right)$ \\
\hline Band Pass Filter & $\mathbf{Y}_{1} \mathbf{Y}_{3} /\left(\mathbf{Y}_{1} \mathbf{Y}_{2}+\mathbf{Y}_{4}\left(\mathbf{Y}_{1}+\mathbf{Y}_{3}\right)\right)$ \\
\hline
\end{tabular}




\begin{tabular}{|c|c|}
\hline \multicolumn{2}{|c|}{ Low Pass Filter } \\
\hline Practical cut-off frequency & $\begin{array}{c}\text { Theoretical cut-off } \\
\text { frequency }\end{array}$ \\
\hline $\mathbf{2 2 . 0 0} \mathbf{~ K H z}$ & $\mathbf{3 2 . 8 0} \mathbf{~ K H z}$ \\
\hline \multicolumn{2}{|c|}{ High Pass Filter } \\
\hline Practical cut-off frequency & $\begin{array}{c}\text { Theoretical cut-off } \\
\text { frequency }\end{array}$ \\
\hline $\mathbf{2 3 . 0 0} \mathbf{~ H z}$ & $\mathbf{2 3 . 2 3} \mathbf{~ K H z}$ \\
\hline
\end{tabular}

\begin{tabular}{|c|c|c|}
\hline \multicolumn{3}{|c|}{ Band Pass Filter } \\
\hline Parameter & $\begin{array}{c}\text { Practical } \\
\text { result }\end{array}$ & Theoretical result \\
\hline $\begin{array}{c}\text { Centre } \\
\text { Frequency(fo) }\end{array}$ & $\mathbf{2 5 . 0 0 ~ K H z}$ & $\mathbf{2 3 . 2 3 ~ K H z}$ \\
\hline Bandwidth & $\mathbf{3 4 . 0 0 ~ K H z}$ & $\mathbf{3 3 . 8 0} \mathbf{~ K H z}$ \\
\hline
\end{tabular}

\section{Conclusion}

Different circuits are implemented successfully using the CFOA. All the circuits are working properly within the Experimental error limits. Circuits implemented have many industrial applications which are discussed in following section.

\section{References}

[1] Franco, S. "Design with operational amplifiers and Analog Integrated Circuits", The Macgraw-Hill Companies, Inc., 668 pages,1998.

[2] Koton, J., Vrba, K. "The design of Frequency filters using autonomous circuits with the complete net of admittances", www.elektorevue.cz,2005.

[3] Ahmed L Soliman, Current Feedback Operational amplifiers based Oscillators, Analog Integrated Circuits and Signal Processing, 23, 45-55, 2000.

[4] Soliman A Mahmoud, Ahmed H Maidan, Ahmed L Soliman, "Low Voltage CMOS Current Feedback Operational Amplifier and its application", ETRI Journal, VOL 29,No 2,2007.

[5] Miss Zin Ma Ma Myo, Dr. Zaw Min Aung, Dr. Zaw Min Naing, "Design and Implementation of Active Band-Pass Filter for Low Frequency RFID System", IMECS, VOL I, 2009.

[6] Firat Kacar, Hakan Kuntman, “CFOA based lossless and lossy Inductance Simulators”, RADIOENGINEERING, VOL 20, No 3, 2011.

[7] Jim Karki, Active Low-Pass Filter Design, Application Report, SLOA049A, Texas Instruments, 2000.

[8] Hung-Yu Wang, Sheng-Hsiung Chang, Tzu-Yi Yang, Po-Yang Tsai, “A Novel Multifunctional CFOA-Based Inverse Filter”, SciRP, $2,2011$. 